\title{
The Significance of Cardiac Arrhythmias in Septic ICU Patients
}

\author{
Daniela lonescu*
}

Department of Anesthesia and Intensive Care, "Iuliu Hatieganu" University of Medicine and Pharmacy, Cluj-Napoca, Romania

The review article published in this issue by Schwartz A et al [1] draws attention to the importance of cardiac arrhythmias and especially that of new-onset atrial fibrillation (AF) and the clinical outcome of septic patients. The incidence of this phenomenon varies in different reports, from $5.8 \%$ [2] to $31-40 \%$ [3-4].

Causes are numerous and different mechanisms have been described in the literature and by the authors of the review. Endotoxin induces tachycardia, increases the cardiac index, and reduces blood pressure and systemic vascular resistance without change in stroke volume [5]. Fluid administration results in a decrease in left ventricular ejection fraction and an increase in ventricular volumes even more than before the administration of fluid therapy [5].

An increased inflammatory response also plays an important role in pathogenesis of cardiac arrhythmias and dysfunction in septic patients. Increased plasma levels of C-reactive protein, IL- 6 and TNF- $\alpha$ may contribute to the onset of AF in septic patients $[1,6]$.

With respect to the pathogenesis of AF in septic patients, the authors describe the involvement of an increased calcium influx through L-type calcium channels and of $\beta$-adrenergic stimulation, either due to an autonomic nervous system dysfunction or due to endogenous/exogenous adrenergic stimulation, leading to an increased opening time of L-type calcium channel. Electrolyte disturbances may play a role in facilitating cardiac arrhythmias. It has been demonstrated that low serum phosphate levels are associated with a greater risk of developing cardiac arrhythmias [7].

The mechanism of global myocardial ischemia has been excluded by studies showing that there are no differences in levels of lactate extraction in patients with septic shock who have myocardial depression as compared to who do not have such depression [5].

The review also proposes clinical strategies in patients with new-onset AF and suspected or confirmed sepsis. The authors suggest that onset of new arrhythmia has a diagnosis value, warranting further investigations related to the probability of on-going sepsis and to ultrasound evaluation for the assessment of a concomitant myocardial dysfunction. Further cultures and antibiotic strategies in the context of "early-goal directed therapy" approach would also be in order. Moreover, the recording of new-onset AF in a septic patient is correlated with mortality and possible additional neurologic events.

The best management of new-onset AF in septic patients is still a matter of debate. Comparisons have been made between $\beta$-blockers, amiodarone, digitalis and electrical conversion, all of which have been used successfully to restore sinus rhythm [8], though most studies indicate that $\beta$-blockers, associated with a better outcome [9], or amiodarone $[8,10]$ noted as being associated with less of a negative inotropic effect, are the best options. Procainamide, magnesium, flecainide, esmolol, verapamil, and diltiazem have also been used in the treatment of AF [9]. The approach rate control versus rhythm control in septic patients favours rhythm control since it was proven that inability to control rhythm is associated with increased mortality [11].

As a conclusion, there are no guidelines and no recommendation can be made regarding the treatment of $\mathrm{AF}$ in septic patients based on the present literature [12].

It is also a matter of debate whether or not fluid therapy is a cause or a treatment of cardiac dysfunctions in sepsis and if large fluid infusions reduces or increases atrial arrhythmias.

In conclusion this review brings to attention the importance of the many unsolved problems associated with cardiac arrhythmias in septic patients. Apart from having some common features with non-septic cardiac patients, new onset AF in septic patients has distinct mechanisms and prognostic values. Well controlled fu- 
ture trials will lead to guidelines for the best treatment of this arrhythmia in septic patients.

\section{REFERENCES}

1. Schwartz A, Brotfain E, Koyfman L, Klein M.Cardiac arrhythmias in septic ICU population: an update. J Crit Care Med. 2015;1:140-6.

2. Christian SA, Schorr C, Ferchau L, Jarbrink ME, Parrillo JE, Gerber DRJ. Clinical characteristics and outcomes of septic patients with new-onset atrial fibrillation. Crit Care. 2008; 23:532-6.

3. Salman S, Bajwa A, Gajic O, Afessa B. Paroxysmal atrial fibrillation in critically ill patients with sepsis. J Intensive Care Med. 2008;23:178-83.

4. Wells GL, Morris PE. Incidence and prognosis of atrial fibrillation in patients with sepsis. Cardiology Research. 2011; 2:293-7.

5. Parrillo JE. Pathogenetic mechanisms of septic shock. N Engl J Med 1993; 328:1471-7.

6. Engelmann MD, Svendsen JH. Inflammation in the genesis and perpetuation of atrial fibrillation. Eur Heart J. 2005; 26:2083-92.
7. Schwartz A, Gurman G, Cohen G, et al. Association between hypophophatemia and cardiac arrhythmias in early stages of sepsis. Eur J Internal Med. 2002;13:434-7.

8. Meierhenrich R, Steinhilber E, Eggermann C, et al. Incidence and prognostic impact of new-onset atrial fibrillation in patients with septic shock: a prospective observational study. Crit Care. 2010;14:108-12.

9. Walkey AJ, Evans SR, Winter MR, Benjamin EJ. Practice patterns and outcomes of treatments for atrial fibrillation during sepsis: a propensity-matched cohort study. Chest. 2015;13:1-10.

10. Trappe HJ, Brandts B, Weismueller P. Arrhythmias in the intensive care patient. Curr Opin Crit Care. 2003;9:345-55.

11. Yoshida T, Fujii T, Uchino S, Takinami M. Epidemiology, prevention, and treatment of new-onset atrial fibrillation in critically ill: a systematic review. Journal of Intensive Care. 2015;3:19.

12. Kanji S, Stewart R, Fergusson DA, Mclntyre L, Turgeon AF, Hébert PC. Treatment of new-onset atrial fibrillation in noncardiac intensive care unit patients: a systematic review of randomized controlled trials. Crit Care Med. 2008;36:1620-4. 